\title{
Coordination of Uncoupled Bimanual Movements by Strictly Timed Interhemispheric Connectivity
}

\author{
Gianpiero Liuzzi, Vanessa Hörniß, Maximo Zimerman, Christian Gerloff, and Friedhelm C. Hummel \\ Brain Imaging and Neurostimulation Laboratory, University Medical Center Hamburg-Eppendorf, Department of Neurology, 20246 Hamburg, Germany
}

Independent use of both hands is characteristic of human action in daily life. By nature, however, in-phase bimanual movements, for example clapping, are easier to accomplish than anti-phase movements, for example playing the piano. It is commonly agreed that interhemispheric interactions play a central role in the coordination of bimanual movements. However, the spatial, temporal, and physiological properties of the interhemispheric signals that coordinate different modes of bimanual movements are still not completely understood. More precisely, do individual interhemispheric connectivity parameters have behavioral relevance for bimanual rapid anti-phase coordination? To address this question, we measured movement-related interhemispheric interactions, i.e., inhibition and facilitation, and correlated them with the performance during bimanual coordination. We found that movement-related facilitation from right premotor to left primary motor cortex (rPMd-lM1) predicted performance in anti-phase bimanual movements. It is of note that only fast facilitation during the preparatory period of a movement was associated with success in anti-phase movements. Modulation of right to left primary motor interaction (rM1-lM1) was not related to anti-phase but predicted bimanual in-phase and unimanual behavior. These data suggest that strictly timed modulation of interhemispheric rPMd-lM1 connectivity is essential for independent highfrequency use of both hands. The rM1-lM1 results indicate that adjustment of connectivity between homologous M1 may be important for the regulation of homologous muscle synergies.

\section{Introduction}

Many human movements in everyday life, such as typing or driving a car, require the suppression of the natural tendency toward bilateral synchronization of the limbs (Varela et al., 2001). Accordingly, one definition of skill is the ability to control the natural tendency of temporally uncoupled (anti-phase) movements toward synchronization, i.e., temporal coupling of both hands (in-phase). Psychophysical evidence has demonstrated that when the frequency of anti-phase movements is increased, the tendency toward symmetry becomes stronger and, finally, results in an unavoidable transition from anti-phase to in-phase (Kelso, 1984; Swinnen, 2002). The involvement of interhemispheric interactions in coordinating bimanual actions has been widely discussed (Geffen et al., 1994). However, the exact mechanisms and pathways by which the brain stabilizes temporally complex bimanual coordination remain elusive.

In particular, the supplementary motor area (SMA) and the premotor cortex have been associated with the coordination of bimanual movements (Brinkman, 1984; Tanji et al., 1988; Hals-

\footnotetext{
Received Jan. 4, 2011; revised March 11, 2011; accepted March 17, 2011.

Author contributions: G.L., C.G., and F.C.H. designed research; G.L., V.H., and M.Z. performed research; G.L., V.H., M.Z., and F.C.H. analyzed data; G.L., C.G., and F.C.H. wrote the paper.

This research was supported by the Deutsche Forschungsgemeinschaft (DFG 0923-102/2-1 to C.G.; DFG LI 1892/ 1-1 to G.L.; www.dfg.de) and by the Forschungsförderungsfonds Medizin of the University of Hamburg (NWF04/07 to F.C.H.; NWF08/07 to G.L.; www.uke.de). We thank the members of the Brain Imaging and Neurostimulation Laboratory for valuable comments on the data and manuscript.

Correspondence should be addressed to Dr. Friedhelm C. Hummel, Brain Imaging and Neurostimulation (BINS) Laboratory, Department of Neurology, University Medical Center Hamburg-Eppendorf, Martinistraße 50, 20246 Hamburg, Germany. E-mail: f.hummel@uke.uni-hamburg.de.

DOI:10.1523/JNEUROSCI.0046-11.2011

Copyright $\odot 2011$ the authors $\quad 0270-6474 / 11 / 319111-07 \$ 15.00 / 0$
}

band et al., 1993). This view has been challenged by evidence in monkeys that showed a subset of neurons within the primary motor cortex (M1) specifically engaged in the control of bimanual movements (Donchin et al., 1998; Gribova et al., 2002). Several neuroimaging studies in humans have demonstrated that anti-phase and in-phase movements share a large core motor network, but additional activations in bihemispheric motor networks have also been detected during anti-phase activities (Sadato et al., 1997; Ehrsson et al., 2002; Ullen et al., 2003; Rocca et al., 2007; Walsh et al., 2008; Meister et al., 2010). In previous studies using functional magnetic resonance imaging (fMRI), brain activity related to transitions from anti-phase to in-phase has been reported to occur in right-lateralized prefrontal, SMA, dorsal premotor (PMd), parietal regions and cerebellum (MeyerLindenberg et al., 2002; Aramaki et al., 2006b). However, when TMS was applied to the different cortical regions previously identified with $\mathrm{fMRI}$, only stimulation to the right PMd substantially perturbed movement coordination and induced by far the most switches from the instable anti-phase to the stable in-phase mode (Meyer-Lindenberg et al., 2002). In a recently published study, an increase of the connectivity profile of the predominantly right parietal, PMd and prefrontal regions within the opposite hemisphere was detected when spatially and temporally uncoupled movements were contrasted with coupled movements (Meister et al., 2010). In contrast, Meister et al. and two additional studies revealed a decrease of connectivity between homologous M1 regions when subjects performed temporally coupled movements of both hands (Grefkes et al., 2008; Maki et al., 2008; Meister et al., 2010).Together, these neuroimaging studies suggest that activity in the right hemisphere, especially in the right PMd, along 
with changes of the connectivity profile across hemispheres play an essential role in the coordination of temporally uncoupled bimanual movements. One central question that still remains open is whether topography and modulation of interhemispheric inhibitory and facilitory circuits are associated with different types of bimanual movements. We thus investigated whether movement-related interhemispheric interactions in distinct parts of the motor cortex are associated with how successfully healthy subjects perform bimanual movements. To this end, the following hypotheses were tested in the present study: (1) right premotor to left primary motor cortex (rPMd-lM1) connectivity contributes to discrete anti-phase and right to left primary motor interaction (rM1-lM1) to in-phase bimanual movements; (2) the temporal evolution of interhemispheric signals; and (3) the extent of inhibition and facilitation between hemispheres are useful parameters to predict how successful (bi)manual coordination is accomplished.

\section{Materials and Methods}

Ethics statement. All subjects gave written informed consent to participate in the experiment according to the Code of Ethics of the World Medical Association (Declaration of Helsinki) before all experimental procedures. The study was approved by the local ethics committee of the University of Hamburg (Hamburg, Germany).

Subjects. Fourteen young healthy right-handed subjects (10 females; mean age, $24.43 \pm 0.69$ years; age range, $22-32$ years) participated in the study. Seven subjects took part in a previous study and were behaviorally tested in the present study (Liuzzi et al., 2010). According to the Edinburgh inventory of handedness (Oldfield, 1971), all subjects were right handed (mean score, $92 \pm 4$ ). They were naive to the experimental purpose of the study and did not play a musical instrument regularly. None of the subjects had a history of serious medical, neurological, or psychiatric illnesses, as probed by a standardized questionnaire.

Experimental protocol. The experimental protocol comprised an investigation of interhemispheric interactions with double-pulse transcranial magnetic stimulation (dpTMS) (see below, Assessment of interhemispheric interactions) and an investigation of bimanual and unimanual coordination (see below, Assessment of bimanual and unimanual performance). The dpTMS experiment served to capture the individual's characteristic of interhemispheric interactions during the initiation of a voluntary movement. To test the association of interhemispheric interactions and behavioral data, we calculated multiple regression analyses to obtain the most effective overall model of physiological and behavioral data (see below, Statistical analysis).

Assessment of interhemispheric interactions. dpTMS is a noninvasive stimulation technique with exceptional temporal resolution. It has been widely used for the investigation of interhemispheric pathways and provides information on effective connectivity, i.e., facilitation or inhibition, between distant brain areas (Hallett, 2000; Walsh and Cowey, 2000; Koch et al., 2006; Duque et al., 2007; Liuzzi et al., 2010). A previous investigation with dpTMS supports the view that during movement preparation the rPMd exerts early and strong interhemispheric facilitation toward the left M1 (lM1), whereas the interaction between homologous M1 (rM1-lM1) is inhibitory and is modulated late during movement preparation (Liuzzi et al., 2010). We adapted this paradigm for the present study.

Before the TMS experiment started, subjects were familiarized with a simple reaction time task. Subjects were seated comfortably in an armchair with hands and forearms resting on a table. They focused on a crosshair displayed centrally on a computer screen $50 \mathrm{~cm}$ in front of the table. Subjects were instructed to perform a brisk right index finger abduction as quickly as possible after the crosshair turned into a Go signal. The stimulus onset asynchrony between appearance of the crosshair and the Go signal varied between 6, 7, and $8 \mathrm{~s}$ in a block-randomized order. To determine the individual reaction time (RT), the average onset of electromyographic (EMG) activity of the right first dorsal interosseus muscle (FDI) was determined in 30 trials. Pairs of Ag/ $\mathrm{AgCl}$ surface electrodes were used in a belly-to-tendon-montage for surface EMG recordings of the right and left FDI. The EMG signal was recorded simultaneously from both FDIs using two separate channels and digitized (sampling rate, $5 \mathrm{kHz}$; bandpass filter, $50 \mathrm{~Hz}$ to $1 \mathrm{kHz}$, CED 1902 amplifier, Cambridge Electronics).

For the ensuing dpTMS experiment, we chose four different time points to determine the time course of interhemispheric interactions during movement preparation: $20,50,80$, and $95 \%$ of the individual RT. In essence, dpTMS provides information on interhemispheric interactions by measuring the influence of a conditioning stimulus (CS) given to one hemisphere over a test stimulus (TS) applied to the opposite hemisphere (interstimulus interval between CS and TS, $10 \mathrm{~ms}$ ). This is a well established procedure to obtain information on interhemispheric interactions during movement preparation (Murase et al., 2004; Duque et al., 2005; Koch et al., 2006; Liuzzi et al., 2010). In the present experiment, two different routes between hemispheres were investigated between right M1 and left M1 (rM1-lM1) and between right PMd and left M1 (rPMd1M1). TMS was delivered by two Magstim 200 connected to two figure eight-shaped coils ( $7 \mathrm{~cm}$ in diameter). Before testing task-related interhemispheric interactions as described above, the optimal location for stimulating the M1 representation of the FDI of both hands ("hot spots") and individual resting motor threshold were determined as described previously (Rossini et al., 1994; Chen et al., 2008). We then determined the stimulation intensities (expressed in percentage of maximum stimulator output) for TS, $\mathrm{CS}_{\mathrm{rPMd}}$, and $\mathrm{CS}_{\mathrm{rM1}}$. TS was adjusted during movement preparation, since motor excitability varies substantially between rest and when cued for a reaction (Chen et al., 1998). Hence, we collected 10 trials of TS alone at $50 \%$ of RT (adjustment run at $t_{(2)}$ ) and repeated the adjustment run to obtain unconditioned motor-evoked potentials (MEPs) of stable average peak-to-peak amplitude of $\sim 1 \mathrm{mV}$. This is an optimal excitability level to induce interhemispheric inhibition (IHI), as demonstrated in a previous study that tested IHI at different intensities of TS (Daskalakis et al., 2002). It has been shown in two previous studies that $\mathrm{rM1}-1 \mathrm{M} 1$ is inhibited during early phases of movement preparation (Murase et al., 2004; Duque et al., 2005). We thus chose a stimulation intensity of $\mathrm{CS}_{\mathrm{rM} 1}$ to reach an inhibition of the conditioned MEP amplitude (CS + TS/TS) corresponding to $50-70 \%$ of the unconditioned MEP amplitude. We collected 10 double-pulse trials of $\mathrm{CS}_{\mathrm{rMl}}$-TS at $50 \%$ of individual RT and repeated the adjustment run to obtain MEPs with a stable average peak-to-peak amplitude of $\sim 0.5$ to $0.7 \mathrm{mV}$. Since we wanted to compare rM1-lM1 directly with $\mathrm{rPMd}-\mathrm{lM} 1$, we adopted the same stimulation intensity of $\mathrm{CS}_{\mathrm{rM} 1}$ for $\mathrm{CS}_{\mathrm{rPMd}}$ to rule out stimulation intensity as a confounder. The TS was always applied over the primary motor representation of the right FDI. To assess interhemispheric interaction between homologous areas of the primary motor cortex (rM11M1), the CS was given over the rM1 of the FDI. To evaluate interhemispheric interactions between the rPMd and the $1 \mathrm{M} 1$ (rPMd$\mathrm{lM} 1$ ), the CS coil was moved $2 \mathrm{~cm}$ anteriorly and $0.5 \mathrm{~cm}$ medially from the right primary motor representation of the FDI. Motor-evoked potentials recorded from the right FDI were used to calculate interhemispheric interactions, defined as conditioned MEP (CS-TS)/unconditioned MEP (only TS) * 100-100. Values above 0 indicate facilitation (conditioned MEP > unconditioned MEP). Values below 0 indicate inhibition (conditioned MEP $<$ unconditioned MEP). For more details please see Liuzzi et al. (2010) and Figure $2 a$, which illustrates the experimental setup together with the results of interhemispheric interactions).

Assessment of bimanual and unimanual performance. Subjects were instructed in a standardized fashion to perform a well evaluated, bimanual, rhythmic finger-tapping task using their index and middle fingers, as described previously (Fig. 1a) (Meyer-Lindenberg et al., 2002; Aramaki et al., 2006b; Serrien, 2008). Two 8-key pads connected to a personal computer were used to record the finger taps. Subjects were instructed to perform discrete anti-phase movements that were defined as the synchronous tapping of left index/right middle finger alternating with the synchronous tapping of left middle/right index finger (Fig. 1a). The tapping frequency was triggered by an auditory metronome that started at 40 beats per minute $(0.67 \mathrm{~Hz})$. Visual cues (start and stop signal, fixation cross) were provided on a 17 inch computer screen by Presentation software (Neurobehavioural Systems), which was also used to record the timing of the key presses. Subjects were instructed to start after the Go signal to look at the fixation cross and to maintain the anti-phase mode 


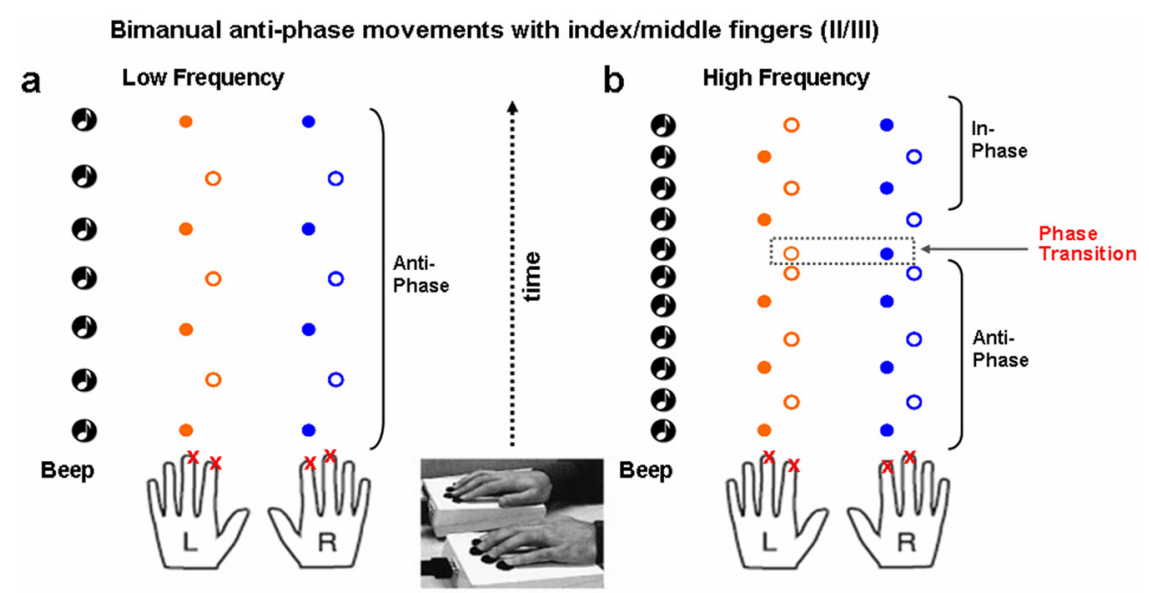

Bimanual in-phase and unimanual control tasks

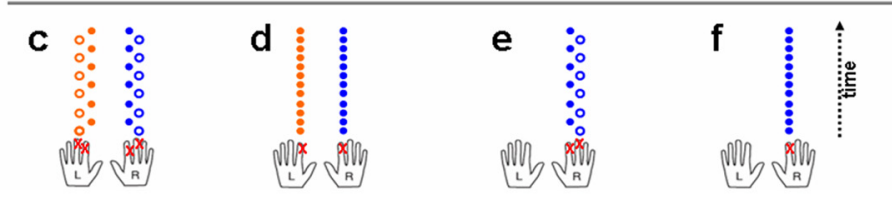

Figure 1. Bimanual and unimanual tasks. $\boldsymbol{a}$, Subjects were instructed to perform bimanual anti-phase movements with index and middle fingers triggered by an auditory metronome. $\boldsymbol{b}$, By consequence of natural coordination constraints, human bimanual movements automatically tend toward symmetry (Kelso, 1984; Meyer-Lindenberg et al., 2002; Swinnen, 2002). Therefore, higher frequency increases task difficulty and results in a phase transition from the anti-phase mode to the in-phase mode (Kelso, 1984; Meyer-Lindenberg et al., 2002; Swinnen, 2002). As a measure of skill, the outcome of this behavioral task was the maximum frequency at which the anti-phase movements could be maintained for $20 \mathrm{~s}$ without switching to in-phase. $c-f$, Control conditions. All control tasks required the same effectors and high speed as the anti-phase task in $\boldsymbol{a}$. Some tasks $(\boldsymbol{c}, \boldsymbol{e})$ required alternating movements between fingers as in $\boldsymbol{a}$, while others only required repetitive movements with the index finger $(\boldsymbol{d}, \boldsymbol{f})$. However, none of the control conditions required anti-phase coupling of the hands. Subjects were instructed to tap as fast as possible for $10 \mathrm{~s}$ bimanually with index and middle fingers in the in-phase mode (c), bimanually with both index fingers simultaneously (d), unimanually alternating index and middle finger (right hand only) $(\boldsymbol{e})$, and with the right index finger only $(\boldsymbol{f})$. As outcome measure for $\mathbf{c} \boldsymbol{f}$, the mean number of taps of three consecutive runs was calculated. Please consult Materials and Methods for details of the behavioral paradigm. L, Left; $R$, right.

for $30 \mathrm{~s}$. Subjects were asked to tap in tune with the auditory metronome. Subjects were instructed to get into the paced rhythm during the first $10 \mathrm{~s}$, which were then discarded from further analysis. The last $20 \mathrm{~s}$ were used to assess accuracy in anti-phase movements. It is known that anti-phase movements are less stable and less accurate than in-phase movements (synchronous tapping of right and left index finger alternating with synchronous tapping of right and left middle finger) and that an increase of frequency ultimately results in a phase transition from the anti-phase to the in-phase mode (Fig. 1a) (Kelso, 1984; Meyer-Lindenberg et al., 2002; Swinnen, 2002). Based on this previously described "egocentric constraint," we titrated the highest frequency at which the anti-phase mode could be maintained for $20 \mathrm{~s}$ in three of four trials without phase transition (Aramaki et al., 2006b). Since it is known that behavioral information such as intention has a modifying influence on phase transitions from unstable to stable movement patterns (Lee et al., 1996; Smethurst and Carson, 2003), all subjects were instructed not to resist a transition, the so-called "do not intervene" condition. Using this approach, a more clear-cut transition from anti-phase to in-phase could be identified in the subjects' behavior, whereas the so-called "stay condition" may lead to phase wandering (Smethurst and Carson, 2003).

To test the association of interhemispheric interactions with symmetrical bimanual movements, we chose bimanual in-phase movements. To this end, we investigated two additional bimanual conditions that do not require anti-phase stability: bimanual in-phase tapping with index and middle fingers and synchronous tapping of both index fingers (Fig. 1c,d). To rule out the possibility that interhemispheric interactions are simply associated with rapid finger movements, be it bimanual or unimanual, we added two unimanual tasks. We tested unimanual alternating tapping of index and middle finger and unimanual tapping of the index fin- ger alone (Fig. 1e,f). For bimanual in-phase and unimanual conditions, subjects were instructed in a standardized fashion to tap as fast as possible after a Go signal for $10 \mathrm{~s}$ until a Stop-signal appeared on the screen. After one familiarization trial, each condition was repeated three times. Each trial was separated by a rest period of $30-60 \mathrm{~s}$. In contrast to antiphase movements, it is known that in-phase movements do not show switches into other stability modes, e.g., into anti-phase (Swinnen, 2002). Thus, the outcome measure that we calculated was the mean number of taps in three runs.

Statistical analysis. Kolmogorov-Smirnov tests for normal distribution were calculated before statistical parametric testing was applied. A two-factorial repeated-measures ANOVA with the factors time (four levels: $t_{(1)}$, $\left.t_{(2)}, t_{(3)}, t_{(4)}\right)$ and location (two levels: rM1lM1, rPMd-lM1) was calculated to evaluate movement-related interhemispheric interactions. Greenhouse-Geisser epsilon determination was used to correct for nonsphericity. To obtain the most effective overall model for the association of interhemispheric interactions with each behavioral task, we performed multiple regression analyses. We used each movement condition separately as the dependent variable (bimanual anti-phase movements of the index/middle fingers, bimanual in-phase tapping with index and middle fingers, synchronous tapping of both index fingers, unimanual alternating tapping of index and middle finger, unimanual tapping of the index finger only) and time-specific "interhemispheric interaction" as the independent variables (rPMd$1 \mathrm{M} 1$ and rM1-lM1 at 20, 50, 80, and 95\% of individual reaction time). Stepwise inclusion/ exclusion of independent variables into the regression model was determined by $F$ probability of $p<0.05$ for inclusion and $p>0.1$ for exclusion. SPSS software (version 15.0.1 for Windows, SPSS) was used for all statistical analyses.

\section{Results}

Interhemispheric interactions

The stimulation intensities for the assessment of interhemispheric interactions during movement preparation were $44.5 \pm 1.38 \%$ of maximum stimulator output for the TS (corresponding to $113.2 \pm$ $3.1 \%$ of the intensity at the individual resting motor threshold) and $41.4 \pm 1.3 \%$ of maximum stimulator output for CS $(110.3 \pm 2.4 \%$ of the intensity at the individual resting motor threshold).

The repeated measures ANOVA of interhemispheric interactions revealed a main effect of location $\left(F_{(1,13)}=22.9 ; p<0.001\right)$ and time $\left(F_{(3,39)}=3.4 ; p=0.034\right)$. Moreover, the interaction of time ${ }^{\star}$ location was significant $\left(F_{(3,39)}=3.0 ; p=0.046\right)($ Fig. $2 b)$. As reported previously (Murase et al., 2004; Duque et al., 2005; Liuzzi et al., 2010), all subjects consistently showed inhibition in rM1-lM1 during early phases of movement preparation, with reduction of inhibition toward movement onset. In line with our previous study (Liuzzi et al., 2010), rPMd-lM1 followed a different temporal pattern compared with rM1-lM1. Connectivity between rPMd-lM1 was already modulated in an early time frame during movement preparation and facilitated contralateral corticospinal output from IM1.

In a nonhierarchical stepwise multiple regression analysis, we then tested whether any of the individual time-specific values of 


\section{a Results of interhemispheric interaction}

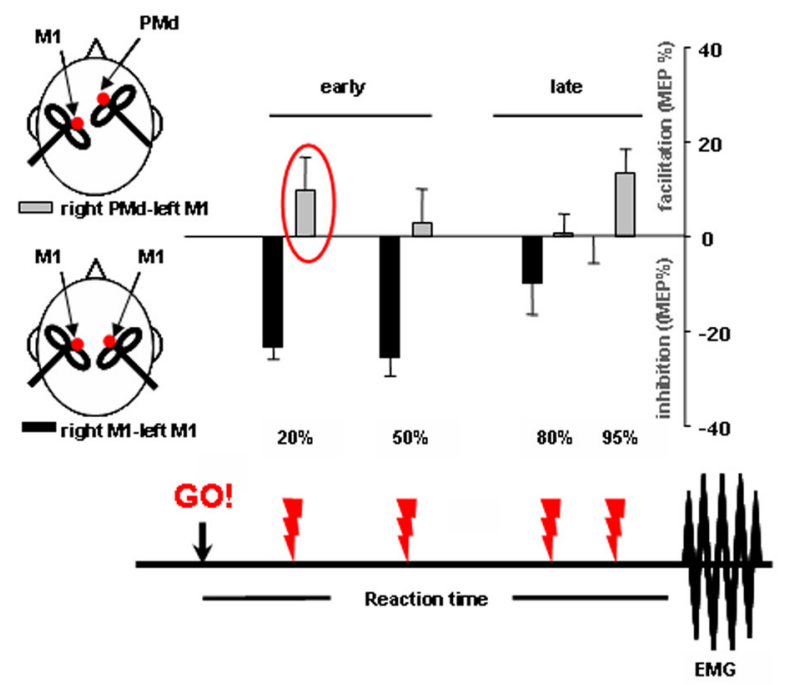

b Multiple regression result of right PMd-left M1 at 20\% of RT versus anti-phase movements IIIIII

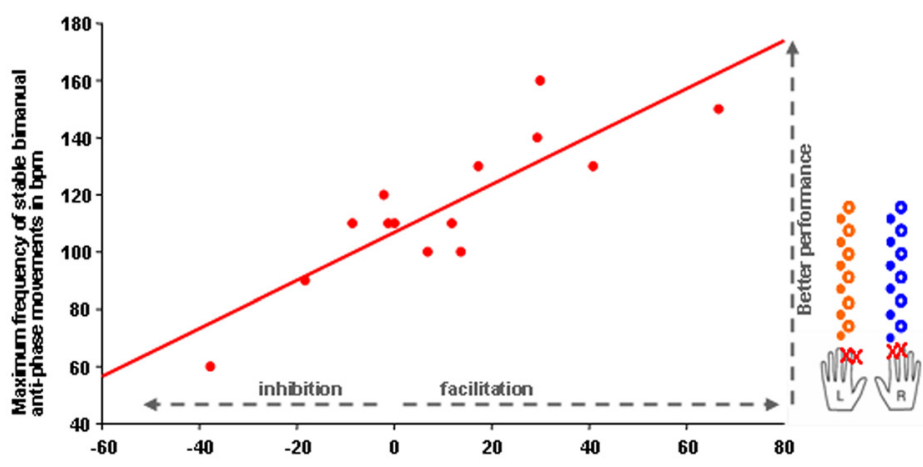

Figure 2. $\quad \boldsymbol{a}$, We measured interhemispheric interactions during movement preparation from the right PMd to the left M1 and between homologous M1 areas of both hemispheres with double-pulse transcranial magnetic stimulation as described previously (Liuzzi et al., 2010). MEPs were recorded from the right index finger early and late during the movement preparation process $(20,50,80$, and $95 \%$ of individual reaction time; for details on the dpTMS methods, see Materials and Methods and Liuzzi et al. (2010)). Group analysis ( $n=14)$ revealed significantly different temporal patterns of rPMd-IM1 versus rM1-IM1 modulation (time * location; $F_{(3,39)}=3.4 ; p=0.034$ ). Using a multiple regression model, we tested the hypothesis of whether any of the individual time-specific values of interhemispheric modulation of rPMd-IM1 and rM1-IM1 explain the variance of performance in maintaining bimanual anti-phase stability. $\boldsymbol{b}, 0 \mathrm{nly}$ rPMd-IM1 interaction at $20 \%$ of RT (red circle in $\boldsymbol{a}$ ) predicted the level of skill in keeping up bimanual anti-phase movements when the index and middle fingers were used ( $p<0.001$ ). In other words, the more the right PMd facilitated corticospinal output from left M1, the faster the subjects performed in the anti-phase task without transition into the in-phase mode.

Table 1. Multiple regression results of interhemispheric interactions and control tasks

\begin{tabular}{|c|c|c|c|c|c|c|c|c|}
\hline \multirow[b]{2}{*}{ Task (dependent variable) } & \multicolumn{2}{|c|}{ Model summary } & \multicolumn{2}{|l|}{ ANOVA } & \multicolumn{4}{|l|}{ Inclusion/exclusion of predictors (dependent variables) } \\
\hline & $R$ & $R^{2}$ & Fvalue & $p$ value & Excluded predictors & Included predictors & $\beta$ value & $p$ value \\
\hline $\begin{array}{l}\text { Biman in-phase } \\
\text { Biman index }\end{array}$ & 0.61 & 0.37 & 5.29 & 0.047 & $\begin{array}{l}\text { rM1-IM1 } t_{(2)}, t_{(3)}, t_{(4)} ; \text { rPMd-IM1 } t_{(1)}, t_{(2)}, t_{(3)}, t_{(4)} \\
\text { All }\end{array}$ & $\begin{array}{l}\text { rM1-IM1 } t_{(1)} \\
\text { None }\end{array}$ & 0.61 & 0.047 \\
\hline Uniman index/middle & 0.72 & 0.52 & 9.82 & 0.012 & rM1-IM1 $t_{(1)}, t_{(2)}, t_{(3)} ;$ rPMd-IM1 $t_{(1)}, t_{(2)}, t_{(3)}, t_{(4)}$ & rM1-IM1 $t_{(4)}$ & -0.72 & 0.012 \\
\hline Uniman index & 0.83 & 0.69 & 19.87 & 0.002 & $\mathrm{rM1-IM1} t_{(1)}, t_{(2)}, t_{(3)} ; \mathrm{rPMd}-\mathrm{IM} 1 t_{(1)}, t_{(2)}, t_{(3)}, t_{(4)}$ & rM1-IM1 $t_{(4)}$ & -0.83 & 0.002 \\
\hline
\end{tabular}

Note that rPMd-IM1 was not associated with any of the control tasks. The significant results of rM1-IM1 associated with the control tasks are given above. $R$ is the multiple correlation coefficient; $R^{2}$ is coefficient of determination (squared $R)$; and $R^{2}$ indicates how much the variance of the dependent variable is explained by the predictors included in the multiple regression. $\beta$ value is the regression coefficient of the included predictor. Values between -1 and 0 indicate a negative relationship of the predictor with the dependent variable. Values between 0 and 1 indicate a positive relationship of the predictor and the dependent variable. Example: the higher the value for rM1-IM1, the higher was the performance for bimanual in-phase tapping. The $p$ value next to the $\beta$ value indicates the statistical significance of the regression related to the included predictor.

interhemispheric modulation between rPMd-lM1 and rM1-lM1 predicted the performance in maintaining anti-phase stability as a measure of higher bimanual skill. All connectivity values (rPMd-lM1 $t_{(1-4)}$ and rM1-lM1 $t_{(1-4)}$ ) were entered as equivalent independent variables in a nonhierarchical multiple regression model. Solely the amount of interhemispheric facilitation from rPMd toward $1 \mathrm{M} 1$ proved to be a predictor for the performance in the bimanual anti-phase task (multiple regression ANOVA: $\left.R^{2}=0.82, F=24.8, p<0.001\right)$. In contrast, none of the interactions between rM1-lM1 was associated with anti-phase performance. In other words, the more rPMd facilitated $1 \mathrm{M} 1$, the higher was the maximum frequency at which the subjects could maintain bimanual anti-phase movements without transition into the easier mirror mode (in-phase) (regression coefficient: $\beta=0.82$, $p<0.001$ ) Fig. $2 b$ ). Note that only the amount of facilitation that occurred early during movement preparation (20\% of reaction time) proved a positive predictor for bimanual anti-phase performance. As another argument in favor of time-specific constraints, late facilitation of interhemispheric premotor-motor connections explained a further part of the behavioral variance but revealed a negative association with anti-phase movements (regression coefficient: $\beta=-0.034, p=0.024$ ). That indicates that subjects who demonstrated late facilitation performed more poorly in bimanual anti-phase movements.

We subsequently asked whether fast modulation of rPMdIM1 is generally beneficial for rapid unimanual and/or bimanual movements or specifically for high-frequency bimanual antiphase movements. To this end, performance in bimanual inphase and unimanual tasks, which also required rapid index/ middle finger movements, was assessed (Fig. $1 c-f$ ). The in-phase task was of particular interest, since the same fingers had to be moved in a quick, alternating manner. Only the coupling mode of both hands was changed. Multiple regression results demonstrated that rPMd-lM1 modulation did not explain variance in the bimanual in-phase or any of the unimanual tasks. On the contrary, modulation of rM1-lM1 significantly contributed to performance in bimanual in-phase and to repetitive unimanual movements (Table 1). Of particular interest, bimanual in-phase performance was associated with the amount of inhibition between rM1-lM1 early during movement preparation $(n=11$; multiple regression: $R^{2}=0.37, F=5.29, p=0.047$ ). Less inhibition between $\mathrm{rM} 1$ and $\mathrm{lM} 1$ was associated with faster bimanual in-phase tapping. 


\section{Discussion}

The need for interhemispheric interactions in coordinating bimanual actions seems obvious and has been confirmed in several previous studies, especially in patients with defects of the corpus callosum (for review, see Geffen et al., 1994). As a physiological measure of interhemispheric interactions, the investigation of interhemispheric connectivity profiles with EEG and fMRI demonstrated stronger interhemispheric coupling of motor regions for anti-phase when compared to in-phase movements (Serrien and Brown, 2002; Serrien, 2008; Meister et al., 2010). However, one open question is whether interhemispheric interactions are predominantly inhibitory, facilitory, or both (Swinnen, 2002; Bloom and Hynd, 2005). The present results suggest that both facilitory and inhibitory interactions between the hemispheres are necessary for bimanual movements. It is likely that there is more than one mode of interhemispheric interaction and that several varieties occur depending on the processing demands of the task (Hellige, 2001). Thus, interhemispheric interactions may be inhibitory and facilitory at different time points during preparation and execution of a motor act. One suggestion is that more complex cognitive tasks rely on excitatory exchange of information between hemispheres (Yazgan et al., 1995). In the present study, we observed both facilitory and inhibitory interhemispheric interactions that were related to different motor regions and to different types of motor coordination. Most strikingly, the amount of rPMd-lM1 facilitation was only related to the performance in the bimanual anti-phase movements, which was the most demanding coordination type of all tested tasks. Moreover, the results indicate that precise timing of facilitation is important for successful anti-phase movements. Early and strong facilitation was associated with better performance in bimanual antiphase movements, whereas delayed facilitation was found in subjects with poor anti-phase stability. In previous studies, it has been demonstrated that while resting hands, interhemispheric PMd-M1 interactions are inhibitory (Mochizuki et al., 2004; Ni et al., 2009) but may turn into strictly timed and short-lasting facilitation during the premovement period (Koch et al., 2006; O'Shea et al., 2007; Liuzzi et al., 2010). By combining TMS and fMRI, Bestmann et al. (2008) showed that a TMS pulse over the PMd elicits different effects on the motor regions of the opposite hemisphere depending on the state of the motor system. During hand grip, TMS led to activity increases in contralateral PMd and M1, whereas activity decreases were observed when the hands were at rest (Bestmann et al., 2008). Thus, PMd appears to exert task-dependent influences over the opposite motor regions. In particular, the rPMd has been suggested to play a specific role in the coordination of bimanual anti-phase movements (MeyerLindenberg et al., 2002; Aramaki et al., 2006b; Meister et al., 2010). The results of the present study suggest that the switch from unstable to stable neural activity during anti-phase is prevented by means of strong and strictly timed facilitation from rPMd toward the opposite primary motor cortex. Considering previous neuroimaging studies together with the present results, the right PMd plays a specific role in stabilizing anti-phase movements. It has been proposed that transfer of visuomotor information from the right to the left hemisphere is faster than the reverse (Marzi et al., 1991). Thus, it is possible that more rapid transmission of signals from right to left hemisphere than in the opposite direction assists the left hemispheric dominance for control of bilateral timing and sequencing (Marzi et al., 1991). If timing and accuracy of responses were predominantly programmed by one hemisphere, bilateral control of the distal limb musculature would still require monitoring activity in the motor regions of the opposite hemisphere. Sending an efference copy of the planned motor program from the nondominant to the dominant hemisphere could be one way to link corollary discharge of both motor cortices, thus allowing for optimal timing of movements in both hands (Geffen et al., 1994). The observation that split-brain patients show enormous difficulty in performing anti-phase bimanual movements underscores the importance of efficient interhemispheric transfer of motor information for bilateral movements (Tuller and Kelso, 1989; Kennerley et al., 2002). As such, fast interhemispheric facilitation may well be one principal means by which the production of bimanual anti-phase movement is kept stable, especially at high speed, when rapid alternating motor commands need to be robustly coordinated across hemispheres. Our findings, however, do not exclude the possibility that several other brain regions are necessary to perform antiphase movements. In addition to rPMd, additional brain regions are engaged in the control of bimanual anti-phase movements. Using fMRI, an increase of activity and connectivity changes in bilateral inferior parietal cortex, basal ganglia, and cerebellum was observed during uncoupled bimanual movements (Meister et al., 2010). It may also be the case that rPMd-lM1 facilitation is just a surrogate parameter that showed an association with behavioral performance in anti-phase movements but may lack a direct causal relationship. However, at least three reasons point to an active involvement of rPMd in stabilizing anti-phase movements. (1) Activity in rPMd was most prominent and most commonly found in several neuroimaging studies (Sadato et al., 1997; Meyer-Lindenberg et al., 2002; Aramaki et al., 2006b; Meister et al., 2010). (2) When TMS was applied to several regions that were observed to be active during anti-phase movements, only stimulation to the rPMd resulted in a breakdown of anti-phase stability (Meyer-Lindenberg et al., 2002). This lateralized effect was confirmed in a more recent study indicating the specific role of the rPMd in anti-phase movements (van den Berg et al., 2010). 3) In the present study, only rPMd-lM1 facilitation, but none of the rM1-lM1 parameters, showed an association with bimanual antiphase performance.

Some authors favor the idea that inhibitory signals are also involved in interhemispheric interactions. The original theories of inhibition by Cook and Kinsbourne postulated that homotopic brain regions are in a steady mutual inhibitory relationship with each other (for review, see Bloom and Hynd, 2005). This assumption is in keeping with more recent neurophysiological studies. Homotopic M1-M1 interaction between representations of distal hand muscles is inhibitory when studied at rest (Ferbert et al., 1992; Gerloff et al., 1998; Daskalakis et al., 2002). However, when an instruction to move the dominant hand is given, inhibition of M1 ipsilateral to the moving hand targeting contralateral M1 is substantially decreased and may turn into a completely disinhibited state immediately before EMG activity appears (Duque et al., 2007; Liuzzi et al., 2010). Interhemispheric inhibition from contralateral M1 targeting ipsilateral M1 displayed a nearly constant inhibition throughout the premovement period (Duque et al., 2007). It has been proposed that these changes in premovement M1-M1 interactions may serve to prevent the appearance of involuntary movements in the resting hand and support dexterity of the moving hand. The results of the present study sustain the assumption that the individual amount of M1-M1 inhibition right before movement onset is related to the speed of repetitive movements with the dominant hand. For bimanual in-phase movements, M1-M1 connectivity studied with functional neuroimaging showed enhanced coupling in both M1 
(Grefkes et al., 2008). However, it is not known so far whether the adjustment of premovement M1-M1 inhibition plays a functional role in bimanual dexterity. In the present study, the maximum speed of bimanual in-phase tapping with the index and middle finger was highest in those subjects that showed weak premovement M1-M1 inhibition. Weak inhibition between homologous M1 representations could be a mechanism to enhance neural crosstalk across hemispheres to propagate synchronous movements of both hands (Aramaki et al., 2006a; Grefkes et al., 2008; Maki et al., 2008). However, the association of premovement M1-M1 inhibition with bimanual in-phase movements was rather weak in the population of the present study. It may well be that the M1-M1 interaction from the dominant to the nondominant hemisphere that was not studied here is more important for bimanual in-phase movements, since activity in the dominant M1 seems to contribute more strongly to the stability of bimanual in-phase movements (Aramaki et al., 2006a; Maki et al., 2008).Together, interhemispheric inhibition or disinhibition may feedforward information to the motor cortex of the other hemisphere when a unimanual or bimanual movement is planned. Interhemispheric inhibition may then serve to prevent mirror movements when a unimanual movement is planned, whereas interhemispheric disinhibition may facilitate bimanual in-phase movements and even transfer motor commands from the dominant to the nondominant hemisphere, allowing for smoother control of bimanual activity (Maki et al., 2008).

It is well known that aging and degenerative brain pathologies like Parkinson's disease (PD) lead to substantial deficits in motor coordination. Aging affects interhemispheric interactions, resulting in reduced M1-M1 inhibition, which is potentially related to the decline of manual dexterity (Talelli et al., 2008). Patients with PD experience great difficulties in bimanual coordination associated with dysfunctions of connectivity in the motor network (Wu et al., 2010). Another recent study in healthy humans demonstrated that applying noninvasive brain stimulation to the PMd induces specific connectivity changes that were related to improvement in the dynamic control of actions (Ward et al., 2010). Taking these recent reports into consideration, our study indicates that dysfunctions in connectivity may depend on the temporal dynamics and the amount of inhibition and facilitation between distant motor cortical regions. Shaping the dynamics of brain connectivity, e.g., with noninvasive brain stimulation, may give rise to new therapeutic avenues for movement disorders caused by malfunctioning interactions between distant brain regions.

\section{References}

Aramaki Y, Honda M, Sadato N (2006a) Suppression of the non-dominant motor cortex during bimanual symmetric finger movement: a functional magnetic resonance imaging study. Neuroscience 141:2147-2153.

Aramaki Y, Honda M, Okada T, Sadato N (2006b) Neural correlates of the spontaneous phase transition during bimanual coordination. Cereb Cortex 16:1338-1348.

Bestmann S, Swayne O, Blankenburg F, Ruff CC, Haggard P, Weiskopf N, Josephs O, Driver J, Rothwell JC, Ward NS (2008) Dorsal premotor cortex exerts state-dependent causal influences on activity in contralateral primary motor and dorsal premotor cortex. Cereb Cortex 18:1281-1291.

Bloom JS, Hynd GW (2005) The role of the corpus callosum in interhemispheric transfer of information: excitation or inhibition? Neuropsychol Rev 15:59-71.

Brinkman C (1984) Supplementary motor area of the monkey's cerebral cortex: short- and long-term deficits after unilateral ablation and the effects of subsequent callosal section. J Neurosci 4:918-929.

Chen R, Yaseen Z, Cohen LG, Hallett M (1998) Time course of corticospinal excitability in reaction time and self-paced movements. Ann Neurol 44:317-325.
Chen R, Cros D, Curra A, Di Lazzaro V, Lefaucheur JP, Magistris MR, Mills K, Rösler KM, Triggs WJ, Ugawa Y, Ziemann U (2008) The clinical diagnostic utility of transcranial magnetic stimulation: report of an IFCN committee. Clin Neurophysiol 119:504-532.

Daskalakis ZJ, Christensen BK, Fitzgerald PB, Roshan L, Chen R (2002) The mechanisms of interhemispheric inhibition in the human motor cortex. J Physiol 543:317-326.

Donchin O, Gribova A, Steinberg O, Bergman H, Vaadia E (1998) Primary motor cortex is involved in bimanual coordination. Nature 395:274-278.

Duque J, Hummel F, Celnik P, Murase N, Mazzocchio R, Cohen LG (2005) Transcallosal inhibition in chronic subcortical stroke. Neuroimage 28:940-946.

Duque J, Murase N, Celnik P, Hummel F, Harris-Love M, Mazzocchio R, Olivier E, Cohen LG (2007) Intermanual differences in movementrelated interhemispheric inhibition. J Cogn Neurosci 19:204-213.

Ehrsson HH, Kuhtz-Buschbeck JP, Forssberg H (2002) Brain regions controlling nonsynergistic versus synergistic movement of the digits: a functional magnetic resonance imaging study. J Neurosci 22:5074-5080.

Ferbert A, Priori A, Rothwell JC, Day BL, Colebatch JG, Marsden CD (1992) Interhemispheric inhibition of the human motor cortex. J Physiol 453:525-546.

Geffen GM, Jones DL, Geffen LB (1994) Interhemispheric control of manual motor activity. Behav Brain Res 64:131-140.

Gerloff C, Cohen LG, Floeter MK, Chen R, Corwell B, Hallett M (1998) Inhibitory influence of the ipsilateral motor cortex on responses to stimulation of the human cortex and pyramidal tract. J Physiol 510:249-259.

Grefkes C, Eickhoff SB, Nowak DA, Dafotakis M, Fink GR (2008) Dynamic intra- and interhemispheric interactions during unilateral and bilateral hand movements assessed with fMRI and DCM. Neuroimage 41:1382-1394.

Gribova A, Donchin O, Bergman H, Vaadia E, Cardoso De Oliveira S (2002) Timing of bimanual movements in human and non-human primates in relation to neuronal activity in primary motor cortex and supplementary motor area. Exp Brain Res 146:322-335.

Hallett M (2000) Transcranial magnetic stimulation and the human brain. Nature 406:147-150.

Halsband U, Ito N, Tanji J, Freund HJ (1993) The role of premotor cortex and the supplementary motor area in the temporal control of movement in man. Brain 116:243-266.

Hellige JB (2001) Hemispheric asymmetry: what's right and what's left. Cambridge, MA: Harvard UP.

Kelso JA (1984) Phase transitions and critical behavior in human bimanual coordination. Am J Physiol 246:R1000-R1004.

Kennerley SW, Diedrichsen J, Hazeltine E, Semjen A, Ivry RB (2002) Callosotomy patients exhibit temporal uncoupling during continuous bimanual movements. Nat Neurosci 5:376-381.

Koch G, Franca M, Del Olmo MF, Cheeran B, Milton R, Alvarez Sauco M, Rothwell JC (2006) Time course of functional connectivity between dorsal premotor and contralateral motor cortex during movement selection. J Neurosci 26:7452-7459.

Lee TD, Blandin Y, Proteau L (1996) Effects of task instructions and oscillation frequency on bimanual coordination. Psychol Res 59:100-106.

Liuzzi G, Hörniss V, Hoppe J, Heise K, Zimerman M, Gerloff C, Hummel FC (2010) Distinct temporospatial interhemispheric interactions in the human primary and premotor cortex during movement preparation. Cereb Cortex 20:1323-1331.

Maki Y, Wong KF, Sugiura M, Ozaki T, Sadato N (2008) Asymmetric control mechanisms of bimanual coordination: an application of directed connectivity analysis to kinematic and functional MRI data. Neuroimage 42:1295-1304.

Marzi CA, Bisiacchi P, Nicoletti R (1991) Is interhemispheric transfer of visuomotor information asymmetric? Evidence from a meta-analysis. Neuropsychologia 29:1163-1177.

Meister IG, Foltys H, Gallea C, Hallett M (2010) How the brain handles temporally uncoupled bimanual movements. Cereb Cortex 20:2996-3004.

Meyer-Lindenberg A, Ziemann U, Hajak G, Cohen L, Berman KF (2002) Transitions between dynamical states of differing stability in the human brain. Proc Natl Acad Sci U S A 99:10948-10953.

Mochizuki H, Huang YZ, Rothwell JC (2004) Interhemispheric interaction between human dorsal premotor and contralateral primary motor cortex. J Physiol 561:331-338. 
Murase N, Duque J, Mazzocchio R, Cohen LG (2004) Influence of interhemispheric interactions on motor function in chronic stroke. Ann Neurol 55:400-409.

Ni Z, Gunraj C, Nelson AJ, Yeh IJ, Castillo G, Hoque T, Chen R (2009) Two phases of interhemispheric inhibition between motor related cortical areas and the primary motor cortex in human. Cereb Cortex 19:1654-1665.

Oldfield RC (1971) The assessment and analysis of handedness: the Edinburgh inventory. Neuropsychologia 9:97-113.

O'Shea J, Sebastian C, Boorman ED, Johansen-Berg H, Rushworth MF (2007) Functional specificity of human premotor-motor cortical interactions during action selection. Eur J Neurosci 26:2085-2095.

Rocca MA, Gatti R, Agosta F, Tortorella P, Riboldi E, Broglia P, Filippi M (2007) Influence of body segment position during in-phase and antiphase hand and foot movements: a kinematic and functional MRI study. Hum Brain Mapp 28:218-227.

Rossini PM, Barker AT, Berardelli A, Caramia MD, Caruso G, Cracco RQ, Dimitrijević MR, Hallett M, Katayama Y, Lücking CH, (1994) Noninvasive electrical and magnetic stimulation of the brain, spinal cord and roots: basic principles and procedures for routine clinical application. Report of an IFCN committee. Electroencephalogr Clin Neurophysiol 91:79-92.

Sadato N, Yonekura Y, Waki A, Yamada H, Ishii Y (1997) Role of the supplementary motor area and the right premotor cortex in the coordination of bimanual finger movements. J Neurosci 17:9667-9674.

Serrien DJ (2008) Coordination constraints during bimanual versus unimanual performance conditions. Neuropsychologia 46:419-425.

Serrien DJ, Brown P (2002) The functional role of interhemispheric synchronization in the control of bimanual timing tasks. Exp Brain Res 147:268-272.

Smethurst CJ, Carson RG (2003) The effect of volition on the stability of bimanual coordination. J Mot Behav 35:309-319.
Swinnen SP (2002) Intermanual coordination: from behavioural principles to neural-network interactions. Nat Rev Neurosci 3:348-359.

Talelli P, Waddingham W, Ewas A, Rothwell JC, Ward NS (2008) The effect of age on task-related modulation of interhemispheric balance. Exp Brain Res 186:59-66.

Tanji J, Okano K, Sato KC (1988) Neuronal activity in cortical motor areas related to ipsilateral, contralateral, and bilateral digit movements of the monkey. J Neurophysiol 60:325-343.

Tuller B, Kelso JA (1989) Environmentally-specified patterns of movement coordination in normal and split-brain subjects. Exp Brain Res 75:306-316.

Ullén F, Forssberg H, Ehrsson HH (2003) Neural networks for the coordination of the hands in time. J Neurophysiol 89:1126-1135.

van den Berg FE, Swinnen SP, Wenderoth N (2010) Hemispheric asymmetries of the premotor cortex are task specific as revealed by disruptive TMS during bimanual versus unimanual movements. Cereb Cortex 20:2842-2851.

Varela F, Lachaux JP, Rodriguez E, Martinerie J (2001) The brainweb: phase synchronization and large-scale integration. Nat Rev Neurosci 2:229-239.

Walsh RR, Small SL, Chen EE, Solodkin A (2008) Network activation during bimanual movements in humans. Neuroimage 43:540-553.

Walsh V, Cowey A (2000) Transcranial magnetic stimulation and cognitive neuroscience. Nat Rev Neurosci 1:73-79.

Ward NS, Bestmann S, Hartwigsen G, Weiss MM, Christensen LO, Frackowiak RS, Rothwell JC, Siebner HR (2010) Low-frequency transcranial magnetic stimulation over left dorsal premotor cortex improves the dynamic control of visuospatially cued actions. J Neurosci 30:9216-9223.

Wu T, Wang L, Hallett M, Li K, Chan P (2010) Neural correlates of bimanual anti-phase and in-phase movements in Parkinson's disease. Brain 133:2394-2409.

Yazgan MY, Wexler BE, Kinsbourne M, Peterson B, Leckman JF (1995) Functional significance of individual variations in callosal area. Neuropsychologia 33:769-779. 